\title{
Habitual and ready positions in female table tennis players and their relation to the prevalence of back pain
}

\author{
Ziemowit Bańkosz \\ Katarzyna Barczyk-Pawelec ${ }^{\text {Equal first author, } 2}$ \\ ${ }^{1}$ Faculty of Sports, Universisty School of Physical Education in Wrocław, Wrocław, Poland \\ ${ }^{2}$ Faculty of Physiotherapy, University School of Physical Education in Wrocław, Wrocław, Poland \\ Corresponding Author: Ziemowit Bańkosz \\ Email address: ziemowit.bankosz@awf.wroc.pl
}

Background The current body of knowledge shows that there is very little research into the occurrence and scale of asymmetry or postural defects in table tennis. It is interesting which regions of the spine are exposed to the greatest changes in the shape of its curvatures and whether the asymmetrical position of the shoulder and pelvic girdles in table tennis players changes when adopting the ready position. Consequently, can overload occur in certain parts of the spine and can the asymmetry deepen as a response of adopting this position? The reply to these questions may be an indication of the need for appropriate compensatory or corrective measures. Therefore, the aim of the study was to evaluate the effect of body position during play on the change in the shape of anteriorposterior spinal curvatures and trunk asymmetry in table tennis players. Methods. To evaluate body posture the photogrammetric method based on the Moiré phenomenon with equipment by CQ Electronic was applied. The study involved 22 female players practicing competitive table tennis (the age of $17 \pm 4.5$, with the average training experience of $7 \pm 4.3$ years, body mass of $47.8 \pm 15.8$, and body height of $161.2 \pm 10.4$ ). Each participant completed an author's own questionnaire on spinal pain. The shape of curvatures in the sagittal and frontal plane was evaluated in the participant in the habitual standing position and in the table tennis ready position. Descriptive statistical analysis was performed and the significance of differences was tested using the Mann-Whitney $U$ test. Results and Conclusions. This study demonstrated the dominance of kyphotic body posture in table tennis players, which can be caused by many hours of using the ready position during playing. After adopting this position, there are significant differences in the angles of anterior and posterior spinal curvatures compared to the habitual posture. This may be the cause of overloads and pain complaints reported by the study participants. Adopting the ready position is also associated with an increase in asymmetry in the position (rotation) of the pelvis and spinous processes (frontal plane). Therefore, training programs should be extended with exercises that relieve the spine in the vertical line and exercises that 
improve symmetry of the work of the upper limbs, body trunk muscles and the pelvis. 


\section{Habitual and ready positions in female table tennis}

\section{2 players and their relation to the prevalence of back}

\section{3 pain}

4 Ziemowit Bańkosz ${ }^{1}$, Katarzyna Barczyk-Pawelec ${ }^{2}$

5

$6 \quad{ }^{1}$ Faculty of Sports, University School of Physical Education in Wrocław, Wrocław, Poland

$7 \quad 2$ Faculty of Physiotherapy, University School of Physical Education in Wrocław, Wrocław,

8 Poland

9

10 Corresponding Author:

11 Ziemowit Bańkosz ${ }^{1}$

12 Paderewskiego 35, Wrocław, 51-612, Poland

13 Email address: ziemowit.bankosz@awf.wroc.pl 
16 Abstract

17 Background The current body of knowledge shows that there is very little research into the occurrence and scale of asymmetry or postural defects in table tennis. It is interesting which regions of the spine are exposed to the greatest changes in the shape of its curvatures and whether the asymmetrical position of the shoulder and pelvic girdles in table tennis players changes when adopting the ready position. Consequently, can overload occur in certain parts of the spine and can the asymmetry deepen as a response of adopting this position? The reply to these questions may be an indication of the need for appropriate compensatory or corrective measures. Therefore, the aim of the study was to evaluate the effect of body position during play on the change in the shape of anterior-posterior spinal curvatures and trunk asymmetry in table tennis players.

Methods. To evaluate body posture the photogrammetric method based on the Moiré phenomenon with equipment by CQ Electronic was applied. The study involved 22 female players practicing competitive table tennis (the age of $17 \pm 4.5$, with the average training experience of $7 \pm 4.3$ years, body mass of $47.8 \pm 15.8$, and body height of $161.2 \pm 10.4)$. Each participant completed an author's own questionnaire on spinal pain. The shape of curvatures in the sagittal and frontal plane was evaluated in the participant in the habitual standing position and in the table tennis ready position. Descriptive statistical analysis was performed and the significance of differences was tested using the Mann-Whitney U test.

Results and Conclusions. This study demonstrated the dominance of kyphotic body posture in table tennis players, which can be caused by many hours of using the ready position during 37 playing. After adopting this position, there are significant differences in the angles of anterior 
38 and posterior spinal curvatures compared to the habitual posture. This may be the cause of

39 overloads and pain complaints reported by the study participants. Adopting the ready position is

40 also associated with an increase in asymmetry in the position (rotation) of the pelvis and spinous

41 processes (frontal plane). Therefore, training programs should be extended with exercises that

42 relieve the spine in the vertical line and exercises that improve symmetry of the work of the

43 upper limbs, body trunk muscles and the pelvis.

\section{Introduction}

46 Table tennis is one of the fastest sports (Kondric et al. 2013). This is mainly due to the short

47 distance between the players (table length is $2.74 \mathrm{~m}$ ) and the speed of the flying ball (up to about

$4840 \mathrm{~m} / \mathrm{s}$ ). For this reason, the players have very little time to react, ranging from 0.2 to 0.4

49 seconds. Except for the service, all player's actions represent the response to the opponent's play.

50 Therefore, each player has to evaluate the parameters of the flying ball: where and how the ball

51 will bounce on the table and what the speed and rotation will be. Then the player must precisely

52 choose the parameters of the stroke such as its type, strength and direction, angle of the racket,

53 place of hitting the ball, and adopt the right position to perform the play. All this causes the

54 player to act in a constant shortage of time. It is therefore essential to remain ready. This is

55 expressed by taking and maintaining the so-called ready position, in which the lower and upper

56 limbs are flexed, the torso is significantly leaned forward, the rocket is kept in front of the

57 player's body, the center of gravity of the body is shifted forward, the body weight is kept on the

58 forefoot, etc. (Fig. 1, Hudetz, 2005).

59 Fig. 1 about here 
60 Another characteristic element of table tennis is the one-sidedness and asymmetry of muscle

61 work because the player plays with one hand. The impact movements are therefore asymmetrical

62 and significantly load one side of the body. Impact movements are characterized by high speed

63 and the impact force is generated based on the principle of "proximal to distal sequence", using

64 the work of the whole body (Iino, \& Kojima, 2009, Bańkosz, \& Winiarski 2017, 2018). The

65 movements of the pelvic girdle, torso and shoulder girdles (Iino, \& Kojima, 2009, 2011, 2016;

66 Bańkosz, \& Winiarski, 2018), especially in the transverse and frontal plane, are of great

67 importance to the achievement of a high impact force.

68 Postural defects (excessive spine curvatures, scoliosis), limb distortions and asymmetry of body

69 build are the factors leading to pain syndromes, degenerative states, disorders of motor functions

70 or even internal organs functioning (Zeyland-Malawka, Prętkiewicz-Abacjew, 2006).

71 Researchers dealing with body posture, the symmetry of body build or proportions of athletes'

72 bodies often find that the risk of occurrence of excessive morphological asymmetry or spinal

73 pain syndromes in athletes is high. Hobbs et al. (2014) have identified a high risk of chronic

74 spinal pain and morphological asymmetry in female and male equestrian athletes. The study

75 found a high correlation between the incidence of injury and certain body mechanics disorders in

76 football players (Watson, 1995). Increased lumbar lordosis and increased or decreased distance

77 between the knees were often associated with muscle strain, while increased thoracic kyphosis

78 and shoulder and trunk asymmetry were associated with back pain. The risk of injury is also high

79 as a result of functional asymmetry, which was found in soccer players (Read et al., 2017).

80 Tiraman and Yaman (2001) demonstrated the relationship between the occurrence of asymmetry

81 in different parts of the body and the occurrence of injuries in adolescents. Krzykała et al.

82 (2018), who examined asymmetry in hockey players, emphasized the role of monitoring of the 
83 magnitude of the asymmetry in preventing injuries and health problems linked to morphological

84 asymmetry. Morton and Callister (2010) found a frequent occurrence of transient abdominal pain

85 after exercise in cases of increased thoracic kyphosis or lumbar lordosis.

86 Many researchers have pointed to the occurrence of asymmetry in athletes. Grabara and Hadzik

87 (2009), assessing the body build of young female and male athletes, found numerous

88 asymmetries with respect to waist triangles and shoulder blade position and tendencies for 89 increased thoracic kyphosis.

90 Grabara (2018) found that thoracic kyphosis increased while lumbar lordosis decreased in young 91 handball players during a two-year training period. A large number of pelvic asymmetries in 92 athletes practising sports with one-sided domination (limb use, rotating upper body) was stressed 93 by Bussey (2010), who examined hockey players, field hockey players, and speed skaters.

94 However, some researchers point out that practicing sport involves correcting or symmetrical 95 development of body posture. Such results have been documented by researchers in the field of 96 taekwondo (Wąsik et al., 2015), gymnastics (Radaŝ, \& Trost Bobiĉ, 2011), or karate(Drzał97 Grabbiec, \& Truszczyńska, 2014). Maloney (2019), in a review of available studies, pointed out 98 that there is no convincing evidence of asymmetry in athletes and that it is sporting activity that 99 can counteract such asymmetries.

100 The current body of knowledge shows that there is very little research into the occurrence and 101 scale of asymmetry or postural defects in table tennis. It is interesting to see how and to what 102 extent the body posture changes during the adoption of a typical playing ready position. To be 103 more specific, the question is which regions of the spine are exposed to the greatest changes in 104 the shape of its curvatures and whether the asymmetrical position of the shoulder and pelvic 105 girdles in table tennis players changes when adopting the ready position? It is also interesting if 
106 table tennis players declare the occurrence of back pain, what are the scale and consequences of

107 this pain and if this pain occurrence is correlated to any of measured body posture parameter?

108 There is no data in the literature concerning this problem. The reply to these questions may be an

109 indication of the need for appropriate compensatory or corrective measures. Therefore, the aims

110 of the study were to: evaluate the effect of body position during play on the change in the shape

111 of anterior-posterior spinal curvatures and trunk asymmetry in table tennis players and to

112 establish the correlation between prevalence of back pain and parameters of body posture in

113 table tennis players.

\section{Materials \& Methods}

116 Participants

117 The method of sampling in the research was judgmental sampling - the research concerned

118 female table tennis players who have been practicing table tennis more than 2 years. The

119 research involved 22 female players practicing competitive table tennis at the age of $17 \pm 4.5$, with

120 the average training experience of $7 \pm 4.3$ years, body weight of $47.8 \pm 15.8$, and body height of

121 161.2 \pm 10.4 . The research was done during afternoon session of training, between 5.00 and 8.00

122 p.m. All participants trained at least 2.5 hours a day 6 times a week, and some of them more

123 often (twice a day). All of them were informed about the research aim and procedures and signed

124 informed consent to participate in the experiment. The research was approved by The Senate's

125 Research Bioethics Commission at the University School of Physical Education in Wrocław.

126 After signing the consent, each participant completed an author's own questionnaire on spinal

127 pain, in which they answered the following questions: (1) How often do you complain about

128 back pain? (never or almost never; rarely; occasionally; often; very often). (2) Which sections of 
129 your back do you find to be the most often painful? (I have no back pain; lumbar spine; thoracic

130 spine; cervical spine; all regions). (3) What is the most frequent pain intensity on a scale from 0

131 to 10 ? (0 - no pain to 10 - unbearable pain). (4) Have you ever had to give up training

132 (competition) because of back pain? (yes, no) (5) Is the spinal pain getting worse? (during

133 training; immediately after the training session, sometime after the training session; no pain). (6)

134 If pain occurs, what is its nature? (radiating to the lower limb; radiating to the upper limb, local

135 without radiation). The answers for the question number 1 helped to divide participants into two

136 groups. Group 1 (8 players) gave answers: never, almost never or rarely and group 2 (14

137 players) gave answers: occasionally; often or very often.

138 Procedures

139 Body posture assessment in all patients was performed with a device for computer analysis of the

140 shape of anterior-posterior curvatures of the spine and trunk asymmetry using the

141 photogrammetric method and a fourth-generation moiré apparatus (CQ Elektronik System,

142 Wroclaw, Poland) that maps the anteroposterior spine curvature (Porto et al. 2010, Barczyk-

143 Pawelec, Sipko 2017) (Fig. 2). The moiré technique is based on a type of optical distortion

144 created by the interference of light waves, as if an image was being refracted. A series of visible

145 lines are projected on the surface of the back, which at different angles are distorted depending

146 on the distance of a given anatomical marker from the projector. In effect, this photogrammetric

147 method mirrors the shape of the back (Fig. 3).

148 Fig. 2 about here

149 Fig. 3 about here

150 Before the examination, the following points were marked on the body of the participant with a

151 washable black marker: spinous processes of spine vertebrae from C7 to S1 and thoracic-lumbar 
152 transition, acromions, inferior angles of scapulae and posterior superior iliac spine. All

153 determinations on the body were made by the same physiotherapist experienced in this type of

154 examination. Based on the contour of the curvature of the spine, the program automatically

155 determined the peaks of thoracic kyphosis and lumbar lordosis. Three-dimensional coordinates

156 of body surface were obtained based on the recorded images of the body trunk of the

157 participants. The parameters determining the anterior-posterior spinal curvatures, the sagittal

158 inclination of the trunk, magnitude of asymmetry within the shoulder and pelvic girdles, and

159 trunk inclination in the frontal plane were calculated.

160 In the sagittal plane, the following angular parameters of spinal curvatures were evaluated and 161 analysed (Fig. 3). :

162 - angle of inclination of the lumbosacral spine $(\alpha)$,

163 - angle of inclination of the thoracolumbar spine ( $\beta)$,

164 - angle of inclination of the upper part of the thoracic region $(\gamma)$,

165 - angle of sagittal inclination of the trunk (KPT). Negative angles indicate the forward

166 inclination of the trunk relative to the vertical line.

167 - angle of thoracic kyphosis (KKP),

$168 \quad-\quad$ angle of lumbar lordosis (KLL)

169 - depth of thoracic kyphosis (GKP)

$170 \quad$ - depth of lumbar lordosis (GLL)

172 Fig 3 about here

173

174 The following body asymmetries were evaluated and analysed in the frontal plane: 
175 (a) Angular parameters (expressed in degrees):

176 - KNT - angle of trunk inclination,

177 - KLB - angle of shoulder line inclination,

178 - KNM - pelvic inclination angle,

179 - KSM - pelvic rotation angle.

180 (b) Length and depth parameters (expressed in $\mathrm{mm}$ ):

181 - UL - difference in the positions of the inferior angles of scapula,

182 - OL- difference in the distance of inferior angles of scapula from the spine,

183 - TT- difference in the height of the waist triangles,

184 - TS- difference in the width of the waist triangles,

185 - UK - deviation of spinous processes from the line of the spine.

186 The magnitude of asymmetry was established on the basis of differences in the placement of

187 osteal points within the trunk. Intervals of these differences were determined for angular and

188 length parameters, distinguishing three levels of asymmetry according to Bibrowicz (Bibrowicz 189 1995).

190 For the angle indices (KNT, KLB, KNM, KSM), it was assumed that:

191 - difference of $0^{\circ}<\mathrm{x} \leq 1.5^{\circ}$ means no asymmetry,

192 - difference of $1.5^{\circ}<x<3^{\circ}$ means moderate asymmetry,

193 - difference of $x \geq 3^{\circ}$ indicates severe asymmetry.

194 For linear asymmetry indices (UL, OL, TT, TS, UK), it has been assumed that:

195 - difference of $0<\mathrm{x} \leq 5 \mathrm{~mm}$ means no asymmetry,

196 - difference of $5<\mathrm{x}<10 \mathrm{~mm}$ means moderate asymmetry,

197 - difference of $x \geq 10$ mm means severe asymmetry. 
198 Body posture was classified on the angular values of the anteroposterior spinal curvatures

199 (compensation index) using the formula $\mu=\gamma-\alpha$, in which $\mu$ was defined as three possible body

200 posture types. The first was a kyphotic-type posture (KT), featuring excess thoracic kyphosis

201 compared with lumbar lordosis in which $\mu>3, \gamma+\beta \geq 29^{\circ}$, and $\alpha+\beta<25^{\circ}$; the second a

202 lordotic'type posture (LT) whereby lumbar lordosis exceeded thoracic kyphosis and $\mu<-3, \alpha+$

$203 \beta \geq 25^{\circ}$, and $\gamma+\beta<29^{\circ}$; and the third was the balanced type (BT) with approximately equal

204 curvatures as defined by $-3 \leq \mu \leq 3$ and $33^{\circ}<\alpha+\beta+\gamma$ (Zeyland-Malawka 1999; Barczyk-

205 Pawelec, \& Sipko. 2017). The shape of curvatures in the sagittal plane was evaluated in the

206 participant in the habitual standing position and in the table tennis ready position after a verbal

207 instruction: "Adopt the ready position!" without giving any additional instructions or guidelines

208 on the quality of the new position. The only thing that the participant could not do was crossing

209 the line determining the distance between the camera and the participant with his or her heels.

210 First of all, body posture was assessed in a habitual standing position without shoes. The test

211 participant stood in a habitual standing position within the field of vision of the camera at a

212 distance of $2.6 \mathrm{~m}$. The participant's feet were positioned on a line parallel to the measurement

213 stand, spaced at the width of the hips. The knee joints were extended and the body weight was

214 evenly distributed on both lower limbs. The upper limbs were placed freely along the torso, the

215 head was positioned freely, and the eyes were looking ahead. After recording the shape of the

216 upper body in the habitual standing position, the examined person, on the instruction of "Adopt

217 the ready position!", adopted the given position and after 5 seconds, another image of the back

218 was recorded.

219 Statistics 
220 The parameters obtained from the examinations were subjected to statistical analysis. Descriptive 221 statistical analysis was performed (normality of distribution was tested by means of the Shapiro222 Wilk test. Means, standard deviations and confidence intervals for mean CI 95\% were calculated 223 for all measured parameters. The significance of differences between habitual and ready

224 positions was tested using the Mann-Whitney U test with the level of statistical significance set 225 at $\mathrm{p} \leq 0.05$, and $\mathrm{d}$-Cohen's effect was calculated. The U Mann-Whitney test was also used to 226 examine differences between body posture parameters of group 1 and 2 . This helped to assess 227 the relation between the parameters of body posture and the frequency of occurrence of pain 228 declared by participants. Statistica 10 package (Statsoft Inc., Tulsa, USA) was used for 229 calculations.

\section{Results}

231 A survey conducted immediately before the examination concerning the incidence of back pain 232 showed that 5 athletes never (or almost never) complained about back pain. Three people 233 complained rarely, 7 - occasionally, 3 - often, and 4 - very often. The thoracic region of the spine 234 was considered to be the most common painful regions of the spine (11 people), followed by the 235 lumbar region ( 8 people) and cervical region ( 2 people). 4 people declared no pain symptoms. 236 The most frequent pain intensity indicated by the respondents (on a scale from 0 - no pain to 10 237 unbearable pain) was 5 ( 6 people), followed by 6 ( 6 people), 4 ( 2 people) and 7 ( 2 people). One 238 person reported the intensity of 8, whereas 4 people - the intensity of 0 . Eight people declared 239 that due to spinal pain they had to stop training (or competition). In 10 people, the pain increased 240 during training, in 4 - immediately after training and in 3 - some time after the training session.

241 In most of the respondents, pain was local, without radiation (13 people). Four people reported 242 pain radiating to the upper limb. 
243 In the sagittal plane, the free posture of table tennis players was characterized by slightly

244 deepened thoracic kyphosis, especially in the upper part. Based on the compensation index, a

245 kyphotic type (KT) of posture was found. The depth of thoracic kyphosis (GKP) was also higher

246 than that of lumbar lordosis (GLL). In the frontal plane, table tennis players were characterized

247 by significant asymmetry, exceeding $10 \mathrm{~mm}$, within the parameters of the difference in height

248 and width of waist triangles (TT and TS) and the difference in the distance between lower

249 shoulder blade angles and spine (OL). Furthermore, table tennis players also showed asymmetry

250 at the pelvic rotation angle in the transverse plane (KSM). The remaining analysed parameters

251 for angular and linear asymmetries (KNT, KLB, UL, KNM, UK) were at a moderate level

252 (difference of $1.5^{\circ}-3^{\circ}$ in case of angles, difference of $5-10 \mathrm{~mm}$ in case of linear measure - see

253 Tab. 1).

254 The change in body position has a significant effect on the angles of anterior-posterior spinal

255 curvatures, the angle of trunk inclination and the depth of thoracic kyphosis and lumbar lordosis

256 (Tab. 1). Significant changes in all three angles of spinal curvatures were observed between the

257 habitual standing position and the ready position. After changing the position from habitual

258 posture to the ready position, the angle of inclination of the upper thoracic and lumbosacral

259 regions increased significantly: their values tripled and the angle of the thoracolumbar region

260 increased more than twice. The value of the angle of trunk inclination (KPT) increased tenfold

261 ( $p<0.01)$. Furthermore, as a result of adopting the ready position, significant changes in the depth

262 of both thoracic kyphosis and lumbar lordosis were observed. In these cases, the effect size

263 calculated based on the d-Cohen test was very high ( $d$-Cohen $\geq 1.0$ ). The change in body position

264 had only a slight effect on the change in the magnitude of asymmetry in body trunk and concerns 
265 mainly pelvic rotation (KSM) and the deviation of the spinous processes (UK). The medium 266 effect size ( $d-C o h e n \geq 0.5)$ was found in this case.

267 Test $\mathrm{U}$ showed also the difference between group 1 and 2 of participants in the case of years of

268 experience - group 1: $3.3 \pm 1.7 \mathrm{y}$ and group $2-9.2 \pm 3.9 \mathrm{y}$, with $\mathrm{p}<0.01$. The significant difference 269 was also indicated in the case of angle $\beta$ in habitual position ( $\mathrm{p}=0.03)$.

270

271 Tab. 1. About here

272 Discussion

273 The aims of the study were to: evaluate the effect of body position during play on the change in 274 the shape of anterior-posterior spinal curvatures and trunk asymmetry in table tennis players and 275 to establish the frequency of occurrence of back pain and its correlation to body posture 276 parameters as well as the scale of back pain in table tennis players. Few scientific studies have 277 analysed the parts of the spine that undergo the greatest changes and their direction. This is of 278 great cognitive importance because table tennis players adopt a specific body position for a long 279 period of time during many hours of training and during the game. Forced positions, i.e. flexion280 based posture adopted during readiness for play, can lead to overloading of the lumbar spine. 281 Kyphotic posture, which was found in our study in table tennis players, may cause various 282 physiological and functional disorders of the player's musculoskeletal system. According to 283 Nachemson (1987), the greatest pressure on the intervertebral discs in the lumbar region (mainly 284 on the 3rd lumbar disc) is observed in the standing position with a simultaneous inclination of 285 the body towards the front, whereas in the habitual standing position, this pressure is almost 2.5 286 times lower. The results of our study showed a significant increase in the value of the angle of 287 trunk inclination when table tennis players adopted the ready position. This angle increased more 
288 than tenfold, hence the pressure on intervertebral discs probably increased, mainly in the lower 289 part of the lumbar spine. The lower part of the lumbar spine (L4-S1) is characterized by greater 290 mobility than its upper part, covering $95 \%$ of its entire range. On the other hand, in the places of 291 the greatest mobility, with additional loads, there are exceptional possibilities of overloading and 292 the appearance of symptoms of overload disease and pain.

293 The problem of overload, spinal pain or risk of injury resulting from faulty posture or 294 morphological asymmetry seems to be common, especially in one-sided and monotypic sports.

295 The main factors of various types of injuries in literature most often mentioned are those 296 resulting from many hours of training and overloads and the specificity of the sport i.e. multiple 297 repetitions that overload specific parts of the body (Saragiotto, Di Pierro, \& Lopes, 2014). 298 The results of our survey confirmed that professional table tennis players experienced spinal 299 pain, with nearly $32 \%$ of the respondents complaining about frequent and very frequent pain 300 occurring mainly during the game or immediately after training. This frequency of pain 301 occurrence is probably correlated with the value of angle of inclination of the thoracolumbar 302 spine ( $\beta)$. As many as $36 \%$ of table tennis players had to stop training because of the pain. 303 Slightly more than half of the respondents estimated the level of pain at the medium level (5-6 on the VAS scale) and 3 persons - at the level of 7-8. This demonstrates that the spine is probably 305 heavily overloaded as a result of many hours of training of this sport, with the majority of the 306 training time based on adopting the position forcing the body to position body trunk at a 307 significant forward inclination, and performing frequent and intensive torsional movements. 308 Apart from the forced forward-leaning position, professional training in table tennis also forces 309 the player to use only one upper limb while playing. Impact movements are very intense, often 310 with the use of submaximal and maximal force, significantly involving the entire body. In order 
311 for the impact force to be maximal, the player must make a rotational movement, depending on

312 the playing limb, from the maximum starting and ending ranges, combined with the transfer of

313 body weight from one lower limb to another, but often with the feet on the ground being locked

314 in one plane. Such forced repetitive movements put strain on the posterior lateral structures of

315 the intervertebral discs, which may result in their damage. The results of our study showed that

316 over $50 \%$ of the respondents complained about local pain, which may suggest that the overload

317 to the perispinal structures is not yet at an advanced stage of the disorder.

318 The results of studies of other authors confirm the frequent occurrence of spinal pains in groups

319 of people practicing various sports (rowers, dancers, fencers, gymnasts, athletes, figure skaters

320 and shooters). They pointed out that this problem was mainly caused by high and substantial

321 workout volumes (Fett et al. 2017; Trompeter et al. 2019). Furthermore, they suggested that

322 training should be monitored by experienced coaches to prevent back pain due to technical errors

323 or too much strain exceeding the training capabilities of young athletes.

324 The training process in table tennis involves daily routines of many hours of exercise (usually

325 from 4 to 6 hours per day), which is observed even in young people at the age of 6 years due to

326 the early specialization. The very young male and female athletes (Harimoto, Ito, Hirano) who

327 are currently in the world's leading position (e.g. https://gossipgist.com/tomokazu-harimoto) are

328 claimed to have started intensive training even at an earlier age. The analysis of the results in our

329 study showed that table tennis players who declared high frequency of occurrence of back pain

330 (Group 2) has been practicing longer than the others (Group 1). It can be assumed that in table

331 tennis an increase of time of sport experience is accompanied by frequency of pain occurrence

332 declared by participants. Taking all the above into account, it can be concluded that the risk of

333 postural defects, spinal pain syndromes or morphological asymmetry exceeding the norm in table 
334 tennis may be high. It is worth noting that in the examined athletes, substantial asymmetry was

335 found in the position of the scapulae and waist, while in other parameters, this asymmetry was at 336 a moderate level.

337 An interesting observation also concerns the transverse and frontal planes. The table tennis 338 players studied showed a greater pelvic torsion after adopting the ready position. This may be 339 due to the specific body arrangement in the ready position, where the player positions the lower 340 limbs asymmetrically, with the foot of the limb opposite to the playing upper limb moved

341 forward. At the same time, it may be a signal that this pelvic asymmetry, which in literature is

342 perceived as a consequence of the domination of one side of the body in sporting activities, may

343 become permanent (Bussey, 2010). Significant asymmetry of pelvic rotation angle was also

344 observed in the group of soccer players (Grabara 2012) and handball players (Grabara 2014). It

345 can be presumed that sport-specific training in asymmetrical sports can lead to asymmetry in the

346 position of the body parts, which over time can be fixed in the habitual position.

347 The study also found an increase in asymmetry within the UK parameter (maximum deflection

348 of spinous process line from the line $\mathrm{C} 7-\mathrm{S} 1$ ) in the frontal plane, which may indicate

349 asymmetrical, unilateral bending of the spine in the ready position. Maintaining such a position

350 for many hours can be conducive to various types of overload and asymmetrical muscle work.

351 Therefore, the practical value of this study may be the observation that training programs should 352 incorporate exercises that relieve the spine in the vertical line and exercises that improve 353 symmetry of the work of the upper limbs, body trunk muscles and the pelvis. The results of our 354 research indicated the need to supplement sports training with physiotherapy methods. These 355 methods should reproduce the lordotic flexion of the spine in relief positions and include 356 exercises to strengthen the postural muscles responsible for proper pelvic anterior tilt. Very 
357 important are also exercises which strengthen the muscles of the torso and upper limb of the non-

358 dominant side and to shape the habit of correct body posture based on the symmetry of the

359 shoulder and hip girdle. Limitation of our study could be some of errors that may appear during

360 measurement, reported in the literature (Mrozkowiak, \& Strzecha, 2012). Another limitation of

361 our work is a relatively small number of participants and a fairly large dispersion (variability) of

362 their age. However, it is not easy to choose a study group consisting of female table tennis

363 players who practice the sport professionally and with a sufficiently long training period. An

364 insignificant asymmetry found in the frontal plane of the study participants (only in the case of

365 the UK and KSM) was also surprising. Our previous research suggested the likelihood of a large

366 asymmetry associated with practicing table tennis, especially in KLB, (Barczyk, Bańkosz,

367 Derlich, 2012). Perhaps the participants of the present study are subjected to corrective and

368 compensatory exercises in the direction that counteracts the asymmetry. The limitation of our

369 study could be also interpretation of magnitude of asymmetry adopted in the research according

370 to Bibrowicz (Bibrowicz, 1995) which was originally designated to children

371

\section{Conclusions}

373 This study demonstrated the dominance of kyphotic body posture in table tennis players., which

374 can be caused by many hours of using the ready position during playing. After adopting this

375 ready position, there are significant differences in the angles of anterior and posterior spinal

376 curvatures compared to the habitual posture. This may be the cause of overloads and pain

377 complaints reported by the study participants. Adopting the ready position is also associated with

378 an increase in asymmetry in the position (transverse palnerotation) of the pelvis and spinous

379 processes (frontal plane). Therefore, training programs should be extended with exercises that 
380 relieve the spine in the vertical line and exercises that improve symmetry of the work of the

381 upper limbs, body trunk muscles and the pelvis. The need to entering compensation and

382 correction programs to a training process confirms frequency of pain occurrence declared by

383 participants which is accompanied by increase of thoracolumbar inclination and time of sport

384 experience

385 References

386 Barczyk-Pawelec K, Sipko T. (2017). Active self-correction of spinal posture in pain-free

387

388

women in response to the command "straighten your back". Women and Health 57(9):1098-1114. doi: 10.1080/03630242.2016.1243605

389

Bibrowicz, K. (1995). Elementy wczesnej diagnostyki bocznych skrzywień kręgosłupa-

390

391

392

393

394

395 asymetria tułowia w płaszczyźnie czołowej. (Elements of early diagnosis of lateral curvatures of the spine - asymmetry of the trunk in the frontal plane, in Polish). Fizjoterapia; 3 (3), 7-15

Bussey, M.D. (2010) Does the demand for asymmetric functional lower body postures in lateral sports relate to structural asymmetry of the pelvis? Journal of Science \& Medicine in Sport 13(3), $360-364$

396

397

398

399

400

401

Drzał-Grabiec, J., \& Truszczyńska, A. (2014). Evaluation of selected postural parameters in children who practice kyokushin karate. Biomedical Human Kinetics, 6, 69-73

Grabara, M. (2018) The posture of adolescent male handball players: A two-year study. Journal of Back and Musculoskeletal Rehabilitation 31, 183-189

Grabara M. (2014). A comparison of the posture between young female handball players and non-traing peers. Journal of Back and Musculoskeletal Rehabilitation 27, 2014, 85-92. 
402 Grabara M. (2012). Analysis of body posture between young football players and their untrained 403 peers. Human Movement 2012, vol. 13(2); 120-126.

404 Grabara, M., \& Hadzik, A. (2009). The body posture in young athletes compared to their peers. 405 Polish Journal of Sports Medicine. 25(2): 115-124.

406 Heneweer H, Vanhees L, Picavet HS.(2009) Physical activity and low back pain: A U-shaped 407 relation? Pain. 2009; 143: 21-25. https://doi.org/10.1016/j.pain.2008.12.033

408 Hudetz, R. (2005). Tenis stołowy 2000 [Table tennis 2000, in polish]. Modest, Łódź, 409 Hobbs, S.J., Baxter, J. Broom, L., Rossell, L.A., Sinclair, J., Clayton, H.M. (2014) Posture, 410 flexibility and grip strength in horse riders, Journal of Human Kinetics, 42, 113-125 DOI: 10.2478/hukin-2014-0066 Section II- Exercise Physiology \& Sports Medicine

412 Krzykała, M.; Leszczyński, P.; Grześkowiak, M.; Podgórski, T.; Woźniewicz-Dobrzyńska, M.; 413 Konarska, A.; Strzelczyk, R.; Lewandowski, J.; Konarski, J.M. (2018). Does field hockey increase morphofunctional asymmetry? A pilot study. HOMO - Journal of Comparative Human Biology. Mar2018, Vol. 69 Issue 1/2, p43-49.

Maloney, SJ. (2018). The relationship between asymmetry and athletic performance: A critical review. Journal of Strength and Conditioning Research 33(9): 2579-2593, 2019

Morton, D.P., Callister, R. (2010) .Influence of posture and body type on the experience of exercise-related transient abdominal pain. Journal of Science and Medicine in Sports

Mrozkowiak, M. \& Strzecha M. (2012). Projection moiré as a modern tool for diagnosis of body 422 posture (in Polish, Abstract in English). Antropomotoryka, 60, 33-47

Nachemson AL. Lumbar intradiscal pressure. In: Jayson MIV (ed.) : The lumbar spine and back pain. Churchill-Livingstone, Edinburgh, London, Melbourne, New York 1987 
425 O'Sullivan PB, Grahamslaw KM, Kendell M, Lapenskie SC, Möller NE, Richards KV. The 426 effect of different standing and sitting postures on trunk muscle activity in a pain-free 427 population. Spine 2002 Jun 1;27(11):1238-44.

428 Porto, F., Gurgel, J., T. Russomano, T., \& T. Farinatti, P.DeT.V. 2010. Moiré topography:

429

430

431

432

433

434

435

436

437

438

439

440

441

442

443

444

445

446

Characteristics and clinical application. Gait \& Posture 32 (3):422-24.

doi:10.1016/j.gaitpost.2010.06.017

Radaŝ, J., Trost Bobiĉ, T (2011). Posture in top-level Croatian rhythmic gymnasts and nontrainees. Kinesiology. 43(1): 64-73

Read, P.J., Oliver, J.L., De Ste Croix, M.B.A., Myer, G.D., Lloyd, R.S. (2017). A prospective investigation to evaluate risk factors for lower extremity injury risk in male youth soccer players. Scandinavian journal of medicine \& science in sports. 28(3):1244-1251

Watson, A.W.S. (1995). Sports injuries in footballers related to defects of posture and body mechanics. Journal of Sports Medicine \& Physical Fitness Dec 1995: Vol. 35 Issue 4. p. 289-294

Wąsik, J, Motow-Czyż, M., Shan, G, Kluszczynski, M. (2015). Comparative analysis of body posture in child and adolescent taekwon-do practitioners and non-practitioners. Ido Movement for Culture. 15(3). 35-40

Wojtys EM, Ashton-Miller JA, Huston LJ, Moga PJ (2000) The association between athletic training time and the sagittal curvature of the immature spine. American Journal of Sports Medicine. 2000 Jul-Aug;28(4):490-8.

Zeylan-Malawka E (1999). Klasyfikacja i ocena postawy ciała w modyfikacji metody Wolańskiego i Nowojorskiego Testu Klasyfikacyjnego.( Classification and assessment of 
447 body posture in the modification of the Wolański and New York Classification Test

448 methods, in Polish) Fizjoterapia. 4 (4). 52-55

449 Zeyland-Malawka, E., Prętkiewicz-Abacjew, (2006). Symptoms of asymmetry in the posture of

450 children and young people -a threat to a fully efficient locomotor system and to health.

$451 \quad$ Nowiny Lekarskie, 75(4): 394-398

452 Żuk, B., Sutkowski, M., Paśko, S., and Grudniewski, T. (2019). Posture correctness of young

453 female soccer players. Sci Rep. 2019; 9: 11179. Published online 2019 Aug 1. doi:

$454 \quad 10.1038 / \mathrm{s} 41598-019-47619-1$

455

456

457 Figure captions

458

459 Fig.1. Ready position

460 Fig. 2. Scheme of the research station

461 Fig. 3. Body posture examination using the photogrammetric method in habitual posture (A) and 462 in the ready position (B)

463

464 


\section{Table $\mathbf{1}$ (on next page)}

Results of examinations in a group of players in the habitual standing position and the ready position: means, standard deviations (SD) and confidence intervals ( $\mathrm{Cl} 95 \%)$, pvalues of the Mann-Whitney U-test and d-Cohen's values

Note: $\alpha$ - angle of inclination of the lumbosacral spine; $\beta$ - angle of inclination of the thoracolumbar spine; $\gamma$ - angle of inclination of the upper part of the thoracic region; $\mathrm{Cl}$ compensation index; KPT - angle of sagittal inclination of the trunk. KKP - angle of thoracic kyphosis, KLL - angle of lumbar lordosis; GKP - depth of thoracic kyphosis; GLL - depth of lumbar lordosis, KNT - angle of trunk inclination; KLB - angle of shoulder line inclination; KNM - pelvic inclination angle; KSM - pelvic rotation angle; UL - difference in the positions of the inferior angles of scapula; OL- difference in the distance of inferior angles of scapulae from the spine; $\pi$-difference in the height of the waist triangles; TS- difference in the width of the waist triangles; UK - deviation of spinous processes from the line of the spine. Differences are significant when $p \leq 0.05$. Effect size is medium when Cohen's $d$ is $0.5 \leq 0.8$ ( $\star$ ) and large when $d \& \gamma \tau ; 0.8(\star \star)$. 
1 Table. 1. Results of examinations in a group of players in the habitual standing position and the ready 2 position: means, standard deviations (SD) and confidence intervals (CI 95\%), p-values of the Mann3 Whitney U-test and d-Cohen's values

4

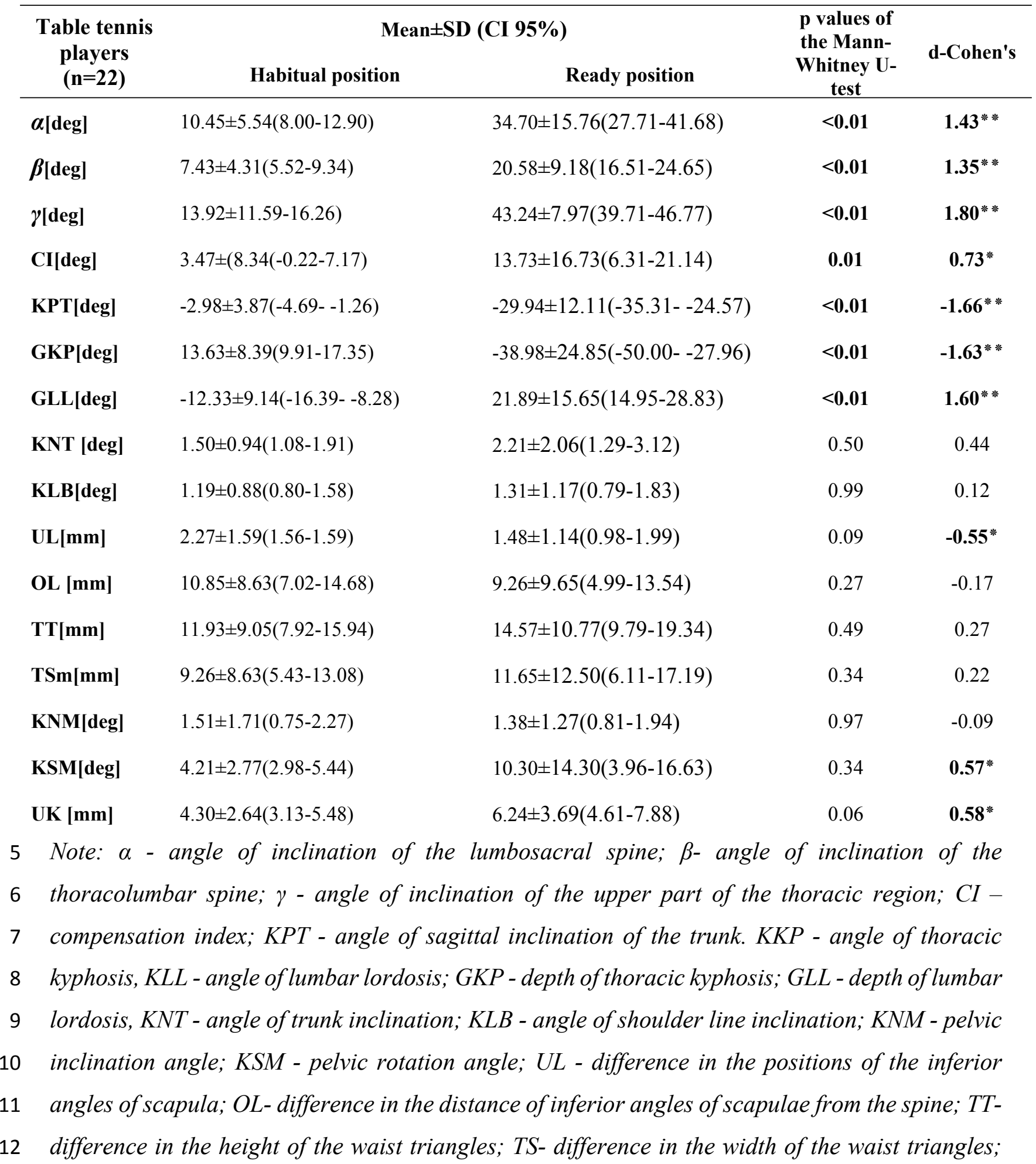


$13 U K$ - deviation of spinous processes from the line of the spine. Differences are significant when $14 p \leq 0.05$. Effect size is medium when Cohen's $d$ is $0.5 \leq 0.8$ (*) and large when $d>0.8$ (**). 15 
Figure 1

Ready position 


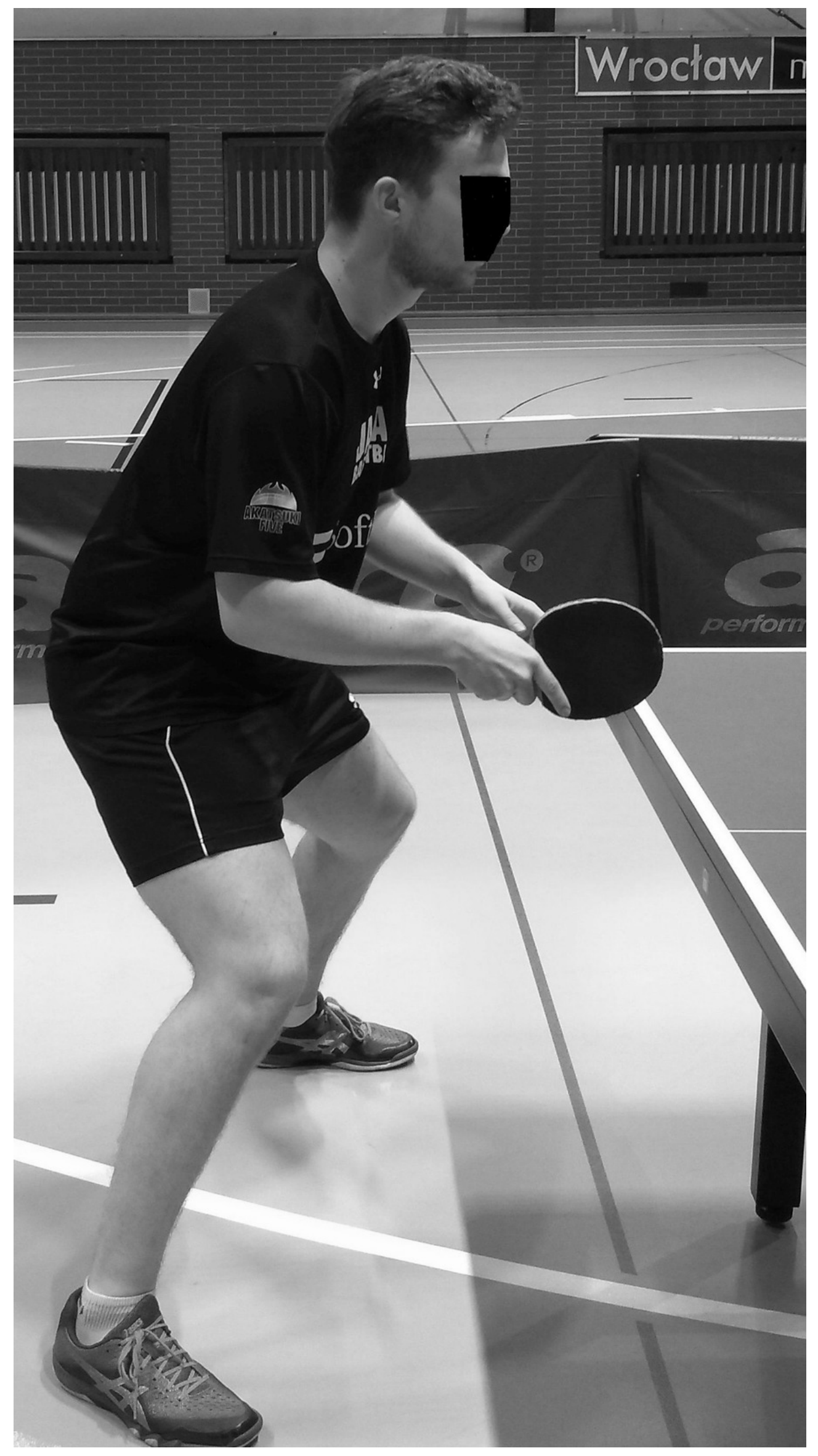


Figure 2

Scheme of the research stand

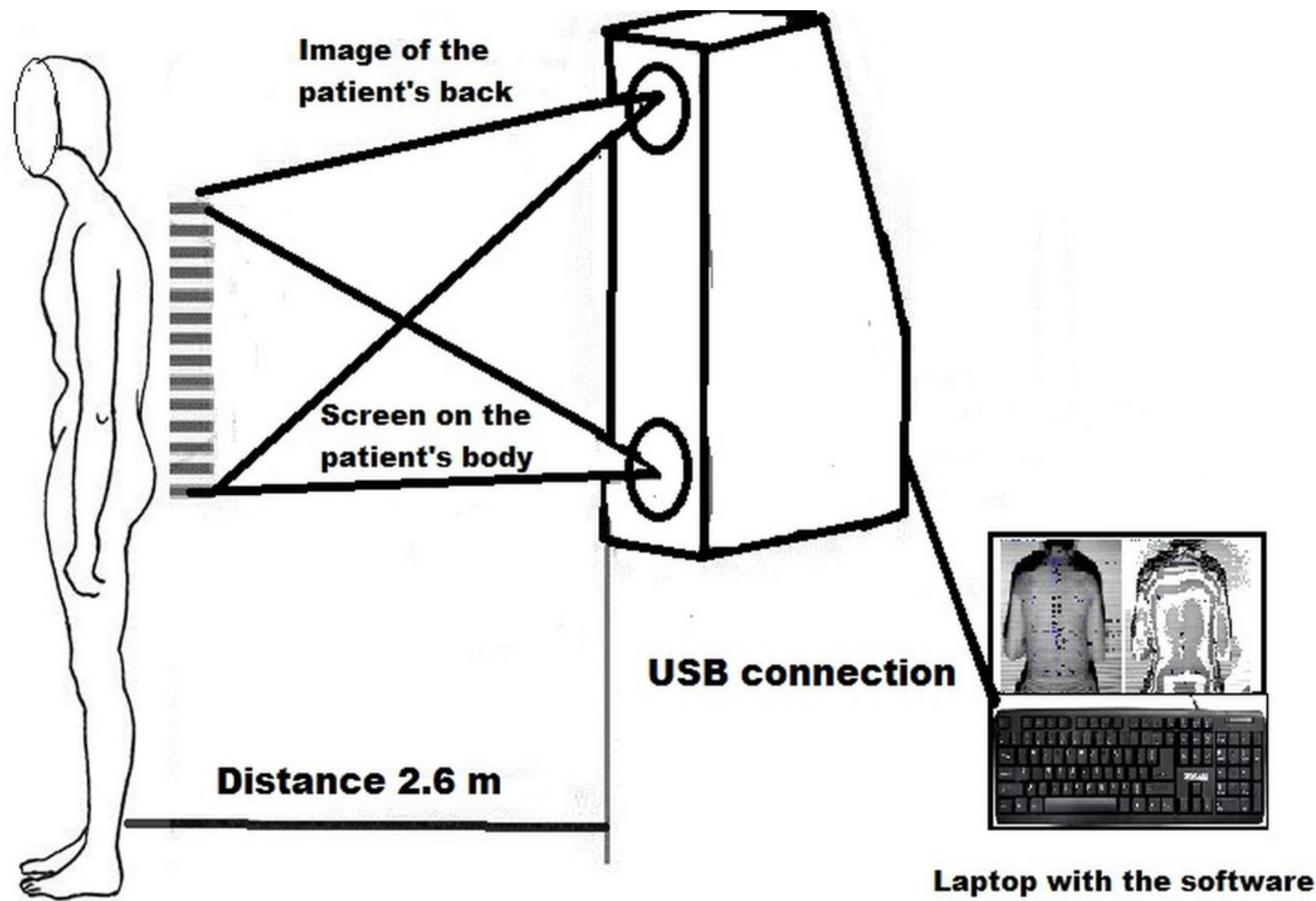


Figure 3

Body posture examination using the photogrammetric method in habitual posture (A) and in the ready position (B)
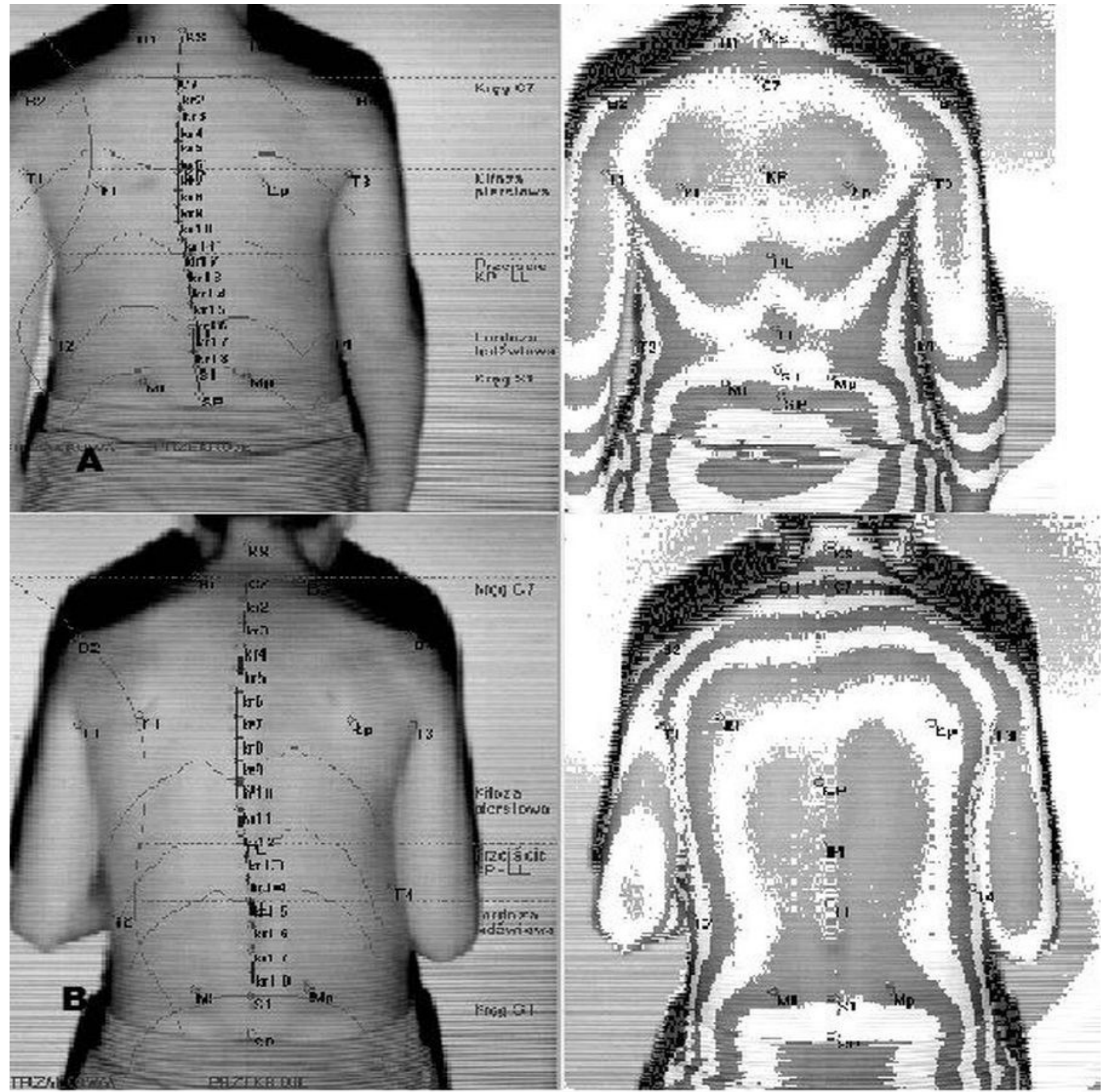